\title{
Several integral inequalities and an upper bound for the bidimensional Hermite-Hadamard inequality
}

Gholamreza Zabandan ${ }^{1}$ and Adem Kılıçman²*

"Correspondence:

akilicman@putra.upm.edu.my ${ }^{2}$ Department of Mathematics and Institute of Mathematical Research, Universiti Putra Malaysia (UPM), Serdang, Selangor 43400 UPM Malaysia

Full list of author information is available at the end of the article

\section{Abstract \\ In this paper we prove several integral inequalities and we find an upper bound of the Hermite-Hadamard inequality for a convex function on a bounded area from the plane in special cases.}

\section{Introduction}

Let $f$ be a convex function on $[a, b]$. Then we have the following inequality, which is called Hermite-Hadamard inequality:

$$
f\left(\frac{a+b}{2}\right) \leq \frac{1}{b-a} \int_{a}^{b} f(x) d x \leq \frac{f(a)+f(b)}{2}
$$

There are many extensions, generalizations and similar results of inequality (1.1). In [1], Fejer established the following weighted generalization of inequality (1.1).

Theorem 1.1 If $:[a, b] \rightarrow \mathbb{R}$ is a convex function, then the inequality

$$
f\left(\frac{a+b}{2}\right) \int_{a}^{b} w(x) d x \leq \int_{a}^{b} f(x) w(x) d x \leq \frac{f(a)+f(b)}{2} \int_{a}^{b} w(x) d x
$$

holds, where $w:[a, b] \rightarrow \mathbb{R}$ is non-negative, integrable and symmetric about $\frac{a+b}{2}$.

In [2], Yang and Tseng proved the following theorem which refines inequality (1.2).

Theorem 1.2 Let $f$ and $w$ be defined as in Theorem 1.1. If $P:[a, b] \rightarrow \mathbb{R}$ is defined by

$$
P(t)=\int_{a}^{b} f\left[t x+(1-t) \frac{a+b}{2}\right] w(x) d x,
$$

then $P$ is convex, increasing on $[0,1]$ and for all $t \in[0,1]$,

$$
f\left(\frac{a+b}{2}\right) \int_{a}^{b} w(x) d x=P(0) \leq P(t) \leq P(1)=\int_{a}^{b} f(x) w(x) d x
$$

O 2013 Zabandan and Klıçman; licensee Springer. This is an Open Access article distributed under the terms of the Creative Commons Attribution License (http://creativecommons.org/licenses/by/2.0), which permits unrestricted use, distribution, and reproduction in any medium, provided the original work is properly cited. 
In this paper, we find an upper bound for $\int_{a}^{b} f(x) g(x) d x$, where $f$ is a convex function on $[a, b]$ and $g$ is non-negative increasing (or decreasing) on $[a, b]$, and $\int_{a}^{b} g(t) d t=1$. Finally, in Section 3 we find an upper bound for the following integral:

$$
\frac{1}{\int_{a}^{b}(g(x)-h(x)) d x} \int_{a}^{b} \int_{h(x)}^{g(x)} F(x, y) d y d x .
$$

\section{Integral inequalities}

Theorem 2.1 Let $f:[a, b] \rightarrow \mathbb{R}$ be a differentiable convex function and $g:[a, b] \rightarrow[0, \infty]$ be a continuous function.

(i) If $g$ is decreasing on $[a, b]$, then

$$
\frac{1}{\int_{a}^{b} g(x) d x} \int_{a}^{b} f(x) g(x) d x \leq \frac{f(a)+f(b)}{2} .
$$

(ii) If $g$ is increasing on $[a, b]$, then

$$
f\left(\frac{a+b}{2}\right) \leq \frac{1}{\int_{a}^{b} g(x) d x} \int_{a}^{b} f(x) g(x) d x .
$$

Proof (i) Denote

$$
H(x)=\int_{a}^{x} f(t) g(t) d t-\frac{1}{2}(f(a)+f(x)) \int_{a}^{x} g(t) d t .
$$

We will show that $H^{\prime}(x) \leq 0$. We have

$$
\begin{aligned}
H^{\prime}(x) & =f(x) g(x)-\frac{1}{2} f^{\prime}(x) \int_{a}^{x} g(t) d t-\frac{1}{2}(f(a)+f(x)) g(x) \\
& =\frac{1}{2}\left[g(x)(f(x)-f(a))-f^{\prime}(x) \int_{a}^{x} g(t) d t\right] .
\end{aligned}
$$

By the extended mean value theorem (Cauchy's theorem), we have

$$
\frac{f(x)-f(a)}{\int_{a}^{x} g(t) d t}=\frac{f^{\prime}(\zeta)}{g(\zeta)} \quad(a<\zeta<x)
$$

On the other hand, by the convexity of $f$ and decreasing of $g$, we obtain

$$
\frac{f(x)-f(a)}{\int_{a}^{x} g(t) d t}=\frac{f^{\prime}(\zeta)}{g(\zeta)} \leq \frac{f^{\prime}(x)}{g(x)}
$$

Since $g$ is non-negative,

$$
H^{\prime}(x)=\frac{1}{2}\left[(f(x)-f(a)) g(x)-f^{\prime}(x) \int_{a}^{x} g(t) d t\right] \leq 0,
$$

which implies that $H$ is decreasing. Hence, $H(b) \leq H(a)=0$. The proof is complete. 
(ii) Denote

$$
H(x)=f\left(\frac{a+x}{2}\right) \int_{a}^{x} g(t) d t-\int_{a}^{x} f(t) g(t) d t
$$

Then we have

$$
\begin{aligned}
H^{\prime}(x) & =\frac{1}{2} f^{\prime}\left(\frac{a+x}{2}\right) \int_{a}^{x} g(t) d t+f\left(\frac{a+x}{2}\right) g(x)-f(x) g(x) \\
& =\frac{1}{2} f^{\prime}\left(\frac{a+x}{2}\right) \int_{a}^{x} g(t) d t-g(x)\left(f(x)-f\left(\frac{a+x}{2}\right)\right) .
\end{aligned}
$$

By the mean value theorem (Lagrange's theorem), there exist $\zeta_{1} \in\left(\frac{a+x}{2}, x\right)$ and $\zeta_{2} \in(a, x)$ such that

$$
\frac{f(x)-f\left(\frac{a+x}{2}\right)}{x-\frac{a+x}{2}}=f^{\prime}\left(\zeta_{1}\right) \text { and } \quad \frac{\int_{a}^{x} g(t) d t-0}{x-a}=g\left(\zeta_{2}\right) .
$$

Hence,

$$
\frac{2\left(f(x)-f\left(\frac{a+x}{2}\right)\right)}{\int_{a}^{x} g(t) d t}=\frac{f^{\prime}\left(\zeta_{1}\right)}{g\left(\zeta_{2}\right)}
$$

By the convexity of $f$ and increasing of $g$, we obtain

$$
\frac{2\left(f(x)-f\left(\frac{a+x}{2}\right)\right)}{\int_{a}^{x} g(t) d t}=\frac{f^{\prime}\left(\zeta_{1}\right)}{g\left(\zeta_{2}\right)} \geq \frac{f^{\prime}\left(\frac{a+x}{2}\right)}{g(x)} .
$$

So,

$$
H^{\prime}(x)=\frac{1}{2} f^{\prime}\left(\frac{a+x}{2}\right) \int_{a}^{x} g(t) d t-g(x)\left(f(x)-f\left(\frac{a+x}{2}\right)\right) \leq 0 .
$$

Therefore, $H$ is decreasing and $H(b) \leq H(a)=0$. The proof is complete.

Theorem 2.2 Let $f:[a, b] \rightarrow \mathbb{R}$ be a convex function and $P:[a, b] \rightarrow[0, \infty)$ be an integrable function such that $\int_{a}^{b} P(x) d x=1$. Then

$$
\int_{a}^{b} f(x) P(x) d x \leq \frac{b f(a)-a f(b)}{b-a}+\frac{f(b)-f(a)}{b-a} \int_{a}^{b} x P(x) d x .
$$

Proof We have

$$
\begin{aligned}
\frac{1}{b-a} \int_{a}^{b} f(x) P(x) d x & =\int_{0}^{1} f(t b+(1-t) a) P(t b+(1-t) a) d t \\
& \leq f(b) \int_{0}^{1} t P(b t+(1-t) a) d t+f(b) \int_{0}^{1}(1-t) P(b t+(1-t) a) d t \\
& =f(b) \int_{a}^{b} \frac{x-a}{b-a} P(x) \frac{d x}{b-a}+f(a) \int_{a}^{b} \frac{b-x}{b-a} P(x) \frac{d x}{b-a}
\end{aligned}
$$




$$
\begin{aligned}
= & \frac{f(b)}{(b-a)^{2}}\left[\int_{a}^{b} x P(x)-a \int_{a}^{b} P(x) d x\right] \\
& +\frac{f(a)}{(b-a)^{2}}\left[b \int_{a}^{b} P(x) d x-\int_{a}^{b} x P(x) d x\right] \\
= & \frac{f(b)}{(b-a)^{2}}\left[\int_{a}^{b} x P(x) d x-a\right]+\frac{f(a)}{(b-a)^{2}}\left[b-\int_{a}^{b} x P(x) d x\right] .
\end{aligned}
$$

So, we get

$$
\int_{a}^{b} f(x) P(x) d x \leq \frac{b f(a)-a f(b)}{b-a}+\frac{f(b)-f(a)}{b-a} \int_{a}^{b} x P(x) d x .
$$

Corollary 2.1 Let $f:[a, b] \rightarrow \mathbb{R}$ be a convex function and $g$ be a non-negative integrable function. Then

$$
\int_{a}^{b} f(x) d x \leq \frac{f(a)+f(b)}{2}
$$

and

$$
\frac{\int_{a}^{b} f(x) g(x) d x}{\int_{a}^{b} g(x) d x} \leq \frac{b f(a)-a f(b)}{b-a}+\frac{f(b)-f(a)}{b-a} \frac{\int_{a}^{b} x g(x) d x}{\int_{a}^{b} g(x) d x} .
$$

The proof is similar to the proof of theorem.

\section{Right bidimensional Hermite-Hadamard inequality}

Let us consider the bidimensional interval $\Delta=[a, b] \times[c, d]$ in $\mathbb{R}^{2}$. Recall that the mapping $f: \Delta \rightarrow \mathbb{R}$ is convex on $\Delta$ if

$$
f(\lambda x+(1-\lambda) z, \lambda y+(1-\lambda) w) \leq \lambda f(x, y)+(1-\lambda) f(z, w)
$$

holds for all $(x, y),(z, w) \in \Delta$ and $\lambda \in[0,1]$. A function $f: \Delta \rightarrow \mathbb{R}$ is called co-ordinated convex on $\triangle$ if the partial mappings $f_{y}:[a, b] \rightarrow \mathbb{R}, f_{y}(u)=f(u, y)$ and $f_{x}:[c, d] \rightarrow \mathbb{R}, f_{x}(v)=$ $f(x, v)$ are convex for all $y \in[c, d]$ and $x \in[a, b]$. Note that every convex function $f: \Delta \rightarrow \mathbb{R}$ is co-ordinated convex, but the converse is not generally true; see [3].

Dragomir in [4] established the following similar inequality of the Hermite-Hadamard inequality for a co-ordinated convex function on a rectangle from the plane $\mathbb{R}^{2}$.

Theorem 3.1 Suppose that $f: \Delta=[a, b] \times[c, d] \rightarrow \mathbb{R}$ is co-ordinated convex on $\Delta$. Then one has the inequalities

$$
\begin{aligned}
f\left(\frac{a+b}{2}, \frac{c+d}{2}\right) & \leq \frac{1}{(b-a)(d-c)} \int_{a}^{b} \int_{c}^{d} f(x, y) d y d x \\
& \leq \frac{f(a, c)+f(a, d)+f(b, c)+f(b, d)}{4}
\end{aligned}
$$

Now, let $\triangle$ be a convex area from the plane $\mathbb{R}^{2}$, bounded by a convex function $y=h(x)$ and a concave function $y=g(x)$ and $x=a, x=b$, such that for any $x \in[a, b], g(x) \geq h(x)$. 
Also, let $F$ be a two-variable convex function on $\triangle$. In [5] and [6], the following inequality is proved:

$$
\begin{aligned}
& \frac{1}{\int_{a}^{b}(g(x)-h(x)) d x} \int_{a}^{b} \int_{h(x)}^{g(x)} F(x, y) d y d x \\
& \geq F\left(\frac{\int_{a}^{b} t(g(t)-h(t)) d t}{\int_{a}^{b}(g(t)-h(t)) d t}, \frac{\frac{1}{2} \int_{a}^{b}\left(g^{2}(t)-h^{2}(t)\right) d t}{\int_{a}^{b}(g(t)-h(t)) d t}\right) .
\end{aligned}
$$

In this paper, we want to find an upper bound for the integral

$$
\frac{1}{\int_{a}^{b}(g(x)-h(x)) d x} \int_{a}^{b} \int_{h(x)}^{g(x)} F(x, y) d y d x .
$$

For this purpose, we reach to the following integral:

$$
\frac{1}{\int_{a}^{b}(g(x)-h(x)) d x} \int_{a}^{b}[F(x, g(x))+F(x, h(x))](g(x)-h(x)) d x .
$$

It is well known that if $F(x, y)$ is increasing relative to $y$ and $y=h(x)$ is convex on $[a, b]$, then $F(x, h(x))$ is convex on $[a, b]$, but we have no information about the convexity of $F(x, h(x))$ generally. So, in special cases, we will find an upper bound for the integral (3.1).

Theorem 3.2 Let $\triangle$ be a bounded area by a convex function $y=h(x)$ and a concave function $y=g(x)$ on $[a, b]$ such that for any $x \in[a, b], g(x) \geq h(x)$ and $g-h$ is increasing on $[a, b]$. Also, let $F$ be a two-variable convex function on $\triangle$ such that $F(x, g(x))$ and $F(x, h(x))$ are convex on $[a, b]$. Then one has the inequality

$$
\begin{aligned}
& \frac{1}{\int_{a}^{b}(g(t)-h(t)) d t} \int_{a}^{b} \int_{h(x)}^{g(x)} F(x, y) d y d x \\
& \quad \leq \frac{1}{4}[F(a, g(a))+F(a, h(a))+F(b, g(b))+F(b, h(b))] .
\end{aligned}
$$

Proof Since $F$ is convex on $\triangle$, hence $F$ is co-ordinated convex on $\triangle$. So, $F_{x}:[h(x), g(x)] \rightarrow$ $\mathbb{R}, F_{x}(y)=F(x, y)$ is convex on $[h(x), g(x)]$ for all $x \in[a, b]$. By the right-hand side of Hermite-Hadamard inequality (1.1), we have

$$
\int_{h(x)}^{g(x)} F(x, y) d y \leq(g(x)-h(x))\left[\frac{F(x, g(x))+F(x, h(x))}{2}\right] .
$$

Integrating this inequality on $[a, b]$, we obtain

$$
\begin{aligned}
& \frac{1}{\int_{a}^{b}(g(t)-h(t)) d t} \int_{a}^{b} \int_{h(x)}^{g(x)} F(x, y) d y d x \\
& \leq \frac{1}{2 \int_{a}^{b}(g(t)-h(t)) d t} \int_{a}^{b}(g(x)-h(x))(F(x, g(x))+F(x, h(x))) d x .
\end{aligned}
$$


Since $g-h$ is increasing and $F(x, g(x)), F(x, h(x))$ are convex on $[a, b]$, by Theorem 2.1(i), we have

$$
\begin{aligned}
& \frac{1}{\int_{a}^{b}(g(t)-h(t)) d t} \int_{a}^{b}(g(x)-h(x))(F(x, g(x))+F(x, h(x))) d x \\
& \quad \leq \frac{1}{2}[F(a, g(a))+F(a, h(a))+F(b, g(b))+F(b, h(b))] .
\end{aligned}
$$

The proof is complete.

Theorem 3.3 Let $\triangle$ be a bounded area by a convex function $h$ and a concave function $g$ on $[a, b]$ such that for any $x \in[a, b], g(x) \geq h(x)$. Also, let $F$ be a two-variable convex function on $\triangle$ such that $F(x, g(x))$ and $F(x, h(x))$ are convex on $[a, b]$. Then one has the inequality

$$
\begin{aligned}
& \frac{1}{\int_{a}^{b}(g(x)-h(x)) d x} \int_{a}^{b} \int_{h(x)}^{g(x)} F(x, y) d y d x \\
& \quad \leq \frac{1}{2}\left[\frac{b-\alpha(b)}{b-a}(F(a, g(a))+F(a, h(a)))+\frac{\alpha(b)-a}{b-a}(F(b, g(b))+F(b, h(b)))\right]
\end{aligned}
$$

where $\alpha(b)=\frac{\int_{a}^{b} t(g(t)-h(t)) d t}{\int_{a}^{b}(g(t)-h(t)) d t}$.

Proof By a similar way to the proof of Theorem 3.2, we have

$$
\begin{aligned}
& \frac{1}{\int_{a}^{b}(g(t)-h(t)) d t} \int_{a}^{b} \int_{h(x)}^{g(x)} F(x, y) d y d x \\
& \leq \frac{1}{2} \frac{1}{\int_{a}^{b}(g(x)-h(x)) d x} \int_{a}^{b}(g(x)-h(x))[F(x, g(x))+F(x, h(x))] d x .
\end{aligned}
$$

Since $F(x, g(x))+F(x, h(x))$ is convex, by Theorem $2.2\left(P(x)=\frac{g(x)-h(x)}{\int_{a}^{b}(g(x)-h(x)) d x}\right)$, we obtain

$$
\begin{aligned}
\frac{1}{2} & \frac{1}{\int_{a}^{b}(g(x)-h(x)) d x} \int_{a}^{b}(g(x)-h(x))[F(x, g(x))+F(x, h(x))] d x \\
\leq & \frac{1}{2} \frac{b[F(a, g(a))+F(a, h(a))]-a[F(b, g(b))+F(b, h(b))]}{b-a} \\
& +\frac{1}{2} \frac{[F(b, g(b))+F(b, h(b))]-[F(a, g(a))+F(a, h(a))]}{b-a} \\
& \times \frac{1}{\int_{a}^{b}(g(x)-h(x)) d x} \int_{a}^{b} x(g(x)-h(x)) d x \\
= & \frac{1}{2}\left[\frac{b-\alpha(b)}{b-a}[F(a, g(a))+F(a, h(a))]+\frac{\alpha(b)-a}{b-a}[F(b, g(b))+F(b, h(b))]\right] .
\end{aligned}
$$

The proof is complete.

In the following theorem, we prove the assertion of Theorem 3.3 with weak conditions. 
Theorem 3.4 Let $\Delta, g$ and $h$ be defined as in Theorem 3.3. Also, let $F$ be a two-variable convex function on $\triangle$ such that

$$
\frac{\partial F(x, g(x))}{\partial g}\left(\frac{g(x)-g(a)}{x-a}-g^{\prime}(x)\right)+\frac{\partial F(x, h(x))}{\partial h}\left(\frac{h(x)-h(a)}{x-a}-h^{\prime}(x)\right) \leq 0,
$$

then we have

$$
\begin{aligned}
& \frac{1}{\int_{a}^{b}(g(x)-h(x)) d x} \int_{a}^{b} \int_{h(x)}^{g(x)} F(x, y) d y d x \\
& \leq \frac{1}{2}\left[\frac{(b-\alpha(b))(F(a, g(a))+F(a, h(a)))+(\alpha(b)-a)(F(b, g(b))+F(b, h(b)))}{b-a}\right],
\end{aligned}
$$

where $\alpha(b)=\frac{\int_{a}^{b} t(g(t)-h(t)) d t}{\int_{a}^{b}(g(t)-h(t))}$.

Proof Denote

$$
H(x)=\int_{a}^{x} \int_{h(x)}^{g(x)} f(t, y) d y d t-\frac{1}{2} K(x) \int_{a}^{x}(g(t)-h(t)) d t,
$$

where

$$
K(x)=\left(\frac{x-\alpha(x)}{x-a}\right)[F(a, g(a))+F(a, h(a))]+\left(\frac{\alpha(x)-a}{x-a}\right)[F(x, g(x))+F(x, h(x))] .
$$

Then we have

$$
H^{\prime}(x)=\int_{h(x)}^{g(x)} F(x, y) d y-\frac{1}{2} K(x)(g(x)-h(x))-\frac{1}{2} K^{\prime}(x) \int_{a}^{x}(g(t)-h(t)) d t .
$$

Since $F$ is convex, so it is co-ordinated convex. Hence, by the right-hand side of the Hermite-Hadamard inequality, we obtain

$$
\begin{aligned}
H^{\prime}(x) \leq & \frac{1}{2}(g(x)-h(x))(F(x, g(x))+F(x, h(x))) \\
& -\frac{1}{2} K(x)(g(x)-h(x))-\frac{1}{2} K^{\prime}(x) \int_{a}^{x}(g(t)-h(t)) d t .
\end{aligned}
$$

So,

$$
H^{\prime}(x) \leq \frac{1}{2}\left[(g(x)-h(x))(F(x, g(x))+F(x, h(x))-K(x))-K^{\prime}(x) \int_{a}^{x}(g(t)-h(t)) d t\right] .
$$

On the other hand, we have

$$
\begin{aligned}
& {\left[\frac{x-\alpha(x)}{x-a} F(a, g(a))+\frac{\alpha(x)-a}{x-a} F(x, h(x))\right]^{\prime}} \\
& \quad=\frac{\left(1-\alpha^{\prime}(x)\right)(x-a)-x+\alpha(x)}{(x-a)^{2}} F(a, g(a)) \\
& \quad+\frac{\alpha^{\prime}(x)(x-a)-\alpha(x)+a}{(x-a)^{2}} F(x, g(x))+F^{\prime}(x, g(x)) \frac{\alpha(x)-a}{x-a} .
\end{aligned}
$$


Now, multiplying each term by

$$
\int_{a}^{x}(g(t)-h(t)) d t
$$

and using the fact

$$
\int_{a}^{x}(g(t)-h(t)) d t \alpha(x)=\int_{a}^{x} t(g(t)-h(t)) d t
$$

we obtain

$$
\int_{a}^{x}(g(t)-h(t)) d t \alpha^{\prime}(x)=(g(x)-h(x))(x-\alpha(x)) .
$$

Therefore,

$$
\begin{aligned}
\int_{a}^{x}(g(t)-h(t)) d t\left[\frac{x-\alpha(x)}{x-a} F(a, g(a))+\frac{\alpha(x)-a}{x-a} F(x, h(x))\right]^{\prime} \\
=\left[-\frac{(g(x)-h(x))(x-\alpha(x))}{x-a}+\frac{\alpha(x)-a}{(x-a)^{2}} \int_{a}^{x}(g(t)-h(t)) d t\right] F(a, g(a)) \\
\quad+\left[\frac{(g(x)-h(x))(x-\alpha(x))}{x-a}+\frac{a-\alpha(x)}{(x-a)^{2}} \int_{a}^{x}(g(t)-h(t)) d t\right] F(x, g(x)) \\
\quad+\int_{a}^{x}(g(t)-h(t)) d t F^{\prime}(x, g(x)) \frac{\alpha(x)-a}{x-a} .
\end{aligned}
$$

By a similar way, we obtain

$$
\begin{aligned}
\int_{a}^{x}(g(t)-h(t)) d t\left[\frac{x-\alpha(x)}{x-a} F(a, h(a))+\frac{\alpha(x)-a}{x-a} F(x, h(x))\right]^{\prime} \\
=\left[-\frac{(g(x)-h(x))(x-\alpha(x))}{x-a}+\frac{\alpha(x)-a}{(x-a)^{2}} \int_{a}^{x}(g(t)-h(t)) d t\right] F(a, h(a)) \\
\quad+\left[\frac{(g(x)-h(x))(x-\alpha(x))}{x-a}+\frac{a-\alpha(x)}{(x-a)^{2}} \int_{a}^{x}(g(t)-h(t)) d t\right] F(x, h(x)) \\
\quad+\int_{a}^{x}(g(t)-h(t)) d t F^{\prime}(x, h(x))\left(\frac{\alpha(x)-a}{x-a}\right) .
\end{aligned}
$$

Thus,

$$
\begin{aligned}
& \int_{a}^{x}(g(t)-h(t)) d t K^{\prime}(x) \\
& =\frac{(g(x)-h(x))(x-\alpha(x))}{x-a}[F(x, g(x))-F(a, g(a))+F(x, h(x))-F(a, h(a))] \\
& \quad-\left(\frac{\alpha(x)-a}{(x-a)^{2}}\right) \int_{a}^{x}(g(t)-h(t)) d t[F(x, g(x))-F(a, g(a))+F(x, h(x))-F(a, h(a))] \\
& \quad+\left(\frac{\alpha(x)-a}{x-a}\right) \int_{a}^{x}(g(t)-h(t)) d t\left[F^{\prime}(x, g(x))+F^{\prime}(x, h(x))\right] .
\end{aligned}
$$


So,

$$
\begin{aligned}
H^{\prime}(x) \leq & \frac{1}{2}\left[(g(x)-h(x))(F(x, g(x))+F(x, h(x))-K(x))-K^{\prime}(x) \int_{a}^{x}(g(t)-h(t)) d t\right] \\
= & \frac{1}{2}(g(x)-h(x)) \frac{x-\alpha(x)}{x-a}[F(x, g(x))+F(x, h(x))-F(a, g(a))-F(a, h(a))] \\
& -\frac{1}{2}\left[\frac{(g(x)-h(x))(x-\alpha(x))}{x-a}-\frac{\alpha(x)-a}{(x-a)^{2}} \int_{a}^{x}(g(t)-h(t)) d t\right] \\
& \times[F(x, g(x))+F(x, h(x))-F(a, g(a))-F(a, h(a))] \\
& -\frac{1}{2} \int_{a}^{x}(g(t)-h(t)) d t\left(\frac{\alpha(x)-a}{x-a}\right)\left(F^{\prime}(x, g(x))+F^{\prime}(x, h(x))\right) .
\end{aligned}
$$

Then it follows that

$$
\begin{aligned}
H^{\prime}(x) \leq & \frac{1}{2}[F(x, g(x))+F(x, h(x))-F(a, g(a))-F(a, h(a))] \\
& \times\left[(g(x)-h(x)) \frac{x-\alpha(x)}{x-a}-\frac{(g(x)-h(x))(x-\alpha(x))}{x-a}\right. \\
& \left.+\frac{\alpha(x)-a}{(x-a)^{2}} \int_{a}^{x}(g(t)-h(t)) d t\right] \\
& -\frac{1}{2} \int_{a}^{x}(g(t)-h(t)) d t\left(\frac{\alpha(x)-a}{x-a}\right)\left(F^{\prime}(x, g(x))+F^{\prime}(x, h(x))\right) .
\end{aligned}
$$

Thus,

$$
\begin{aligned}
H^{\prime}(x) \leq & \frac{1}{2}\left(\frac{\alpha(x)-a}{x-a}\right) \int_{a}^{x}(g(t)-h(t)) d t \\
& \times\left[\frac{F(x, g(x))-F(a, g(a))}{x-a}+\frac{F(x, h(x))-F(x, g(x))}{x-a}-F^{\prime}(x, g(x))-F^{\prime}(x, h(x))\right] .
\end{aligned}
$$

Now, notice that if $F(x, g(x)), F(x, h(x))$ were convex on $[a, b]$, we can deduce the assertion of Theorem 3.3. Since $F$ is convex on $\triangle$, we have

$$
F(x, g(x))-F(a, g(a)) \leq \frac{\partial F(x, g(x))}{\partial x}(x-a)+\frac{\partial F(x, g(x))}{\partial g}(g(x)-g(a))
$$

or

$$
\begin{aligned}
& \frac{F(x, g(x))-F(a, g(a))}{x-a} \\
& \quad \leq \frac{\partial F(x, g(x))}{\partial x}+\frac{\partial F(x, g(x))}{\partial g} \frac{(g(x)-g(a))}{x-a} .
\end{aligned}
$$

By a similar way, we have

$$
\begin{aligned}
& \frac{F(x, h(x))-F(a, h(a))}{x-a} \\
& \quad \leq \frac{\partial F(x, h(x))}{\partial x}+\frac{\partial F(x, h(x))}{\partial h} \frac{h(x)-h(a)}{x-a} .
\end{aligned}
$$


Note that

$$
F^{\prime}(x, g(x))=\frac{\partial F(x, g(x))}{\partial x}+\frac{\partial F(x, g(x))}{\partial g} g^{\prime}(x)
$$

and

$$
F^{\prime}(x, h(x))=\frac{\partial F(x, h(x))}{\partial x}+\frac{\partial F(x, h(x))}{\partial h} h^{\prime}(x) .
$$

So,

$$
\begin{aligned}
H^{\prime}(x) \leq & \frac{1}{2} \frac{\alpha(x)-a}{x-a} \int_{a}^{x}(g(t)-h(t)) d t\left[\frac{\partial F(x, g(x))}{\partial x}\right. \\
& +\frac{\partial F(x, g(x))}{\partial g} \frac{g(x)-g(a)}{x-a}+\frac{\partial F(x, h(x))}{\partial x} \\
& +\frac{\partial F(x, h(x))}{\partial h} \frac{h(x)-h(a)}{x-a}-\frac{\partial F(x, g(x))}{\partial x} \\
& \left.-\frac{\partial F(x, g(x))}{\partial g} g^{\prime}(x)-\frac{\partial F(x, h(x))}{\partial x}-\frac{\partial F(x, h(x))}{\partial h} h^{\prime}(x)\right] .
\end{aligned}
$$

Thus,

$$
\begin{aligned}
H^{\prime}(x) \leq & \frac{1}{2} \frac{\alpha(x)-a}{x-a} \int_{a}^{x}(g(t)-h(t)) d t\left[\frac{\partial F(x, g(x))}{\partial g}\left(\frac{g(x)-g(a)}{x-a}-g^{\prime}(x)\right)\right. \\
& \left.+\frac{\partial F(x, h(x))}{\partial x}\left(\frac{h(x)-h(a)}{x-a}-h^{\prime}(x)\right)\right] \leq 0 .
\end{aligned}
$$

Note that $\alpha(x) \geq a$. Therefore, $H$ is decreasing and

$$
H(b) \leq H(a)=0 .
$$

The proof is complete.

Remark 3.1 Notice that since $g$ is concave and $h$ is convex on $[a, b]$, so $g^{\prime}$ is decreasing and $h^{\prime}$ is increasing on $[a, b]$. By the mean value theorem, we have

$$
\frac{g(x)-g(a)}{x-a}-g^{\prime}(x) \geq 0 \quad \text { and } \quad \frac{h(x)-h(a)}{x-a}-h^{\prime}(x) \leq 0 .
$$

In particular, if we have $g(x)=m x+n$, then $\frac{g(x)-g(a)}{x-a}-g^{\prime}(x)=0$. So, if $\frac{\partial F(x, h(x))}{\partial h} \geq 0$, then

$$
\begin{aligned}
& \frac{\partial F(x, g(x))}{\partial g}\left[\frac{g(x)-g(a)}{x-a}-g^{\prime}(x)\right]+\frac{\partial F(x, h(x))}{\partial x}\left[\frac{h(x)-h(a)}{x-a}-h^{\prime}(x)\right] \\
& \quad=\frac{\partial F(x, h(x))}{\partial x}\left[\frac{h(x)-h(a)}{x-a}-h^{\prime}(x)\right] \leq 0 .
\end{aligned}
$$

In the following theorem, we find an upper bound of the Hermite-Hadamard inequality for a co-ordinated convex function. 
Theorem 3.5 Let $\triangle, g$ and $h$ be defined as in Theorem 3.3. Also, let $F$ be a convex function only relative to $y$, that is, $F_{x}:[h(x), g(x)] \rightarrow \mathbb{R}, F_{x}(v)=F(x, v)$ is convex for all $x \in[a, b]$. If $F^{\prime}(x, g(x))+F^{\prime}(x, h(x)) \geq 0$, then

$$
\frac{1}{\int_{a}^{b}(g(t)-h(t)) d t} \int_{a}^{b} \int_{h(x)}^{g(x)} F(x, y) d y d x \leq \frac{1}{2}[F(b, g(b))+F(b, h(b))] .
$$

Proof Denote

$$
H(x)=\int_{a}^{x} \int_{h(x)}^{g(x)} F(t, y) d y d t-\frac{1}{2} \int_{a}^{x}(g(t)-h(t)) d t[F(x, g(x))+F(x, h(x))] .
$$

We have

$$
\begin{aligned}
H^{\prime}(x)= & \int_{h(x)}^{g(x)} F(x, y) d y-\frac{1}{2}(g(x)-h(x))(F(x, g(x))+F(x, h(x))) \\
& -\frac{1}{2} \int_{a}^{x}(g(t)-h(t)) d t\left(F^{\prime}(x, g(x))+F^{\prime}(x, h(x))\right) .
\end{aligned}
$$

Since $F$ is convex relative to $y$, by the right-hand side of the Hermite-Hadamard inequality, we obtain

$$
\begin{aligned}
H^{\prime}(x) \leq & \frac{1}{2}(g(x)-h(x))(F(x, g(x))+F(x, h(x))) \\
& -\frac{1}{2}(g(x)-h(x))(F(x, g(x))+F(x, h(x))) \\
& -\frac{1}{2} \int_{a}^{x}(g(t)-h(t)) d t\left(F^{\prime}(x, g(x))+F^{\prime}(x, h(x))\right) \\
= & -\frac{1}{2} \int_{a}^{x}(g(t)-h(t)) d t\left(F^{\prime}(x, g(x))+F^{\prime}(x, h(x))\right) \\
\leq & 0 .
\end{aligned}
$$

So, $H$ is decreasing on $[a, b]$. That is, $H(b) \leq H(a)=0$.

\section{Examples}

Example 4.1 Let $F(x, y)=x^{2}+y^{2}$ and $\triangle$ be bounded by $g(x)=\sqrt{1-x^{2}}, h(x)=x-1$ on $[0,1]$. Then $g(x)-h(x)=\sqrt{1-x^{2}}-x+1$ is decreasing on $[0,1]$ and $F(x, g(x))=1, F(x, h(x))=$ $x^{2}+(x-1)^{2}$ are convex on $[0,1]$. By Theorem 3.2 , we have

$$
\begin{aligned}
& \frac{1}{\int_{0}^{1}\left(\sqrt{1-x^{2}}-x+1\right) d x} \int_{0}^{1} \int_{x-1}^{\sqrt{1-x^{2}}}\left(x^{2}+y^{2}\right) d y d x \\
& \leq \frac{1}{4}[F(0, g(0))+F(0, h(0))+F(1, g(1))+F(1, h(1))] .
\end{aligned}
$$

By easy calculation, we see that

$$
\int_{0}^{1}\left(\sqrt{1-x^{2}}-x+1\right) d x=\frac{\pi}{4}+\frac{1}{2}=\frac{\pi+2}{4}
$$


and

$$
\frac{4}{\pi+2} \int_{0}^{1} \int_{x-1}^{\sqrt{1-x^{2}}}\left(x^{2}+y^{2}\right) d y d x \leq 1
$$

Example 4.2 Let $F, g$ and $h$ be defined as in Example 4.1. By Theorem 3.3, we have

$$
\begin{aligned}
\alpha(1) & =\frac{\int_{0}^{1} t\left(\sqrt{1-t^{2}}-t+1\right) d t}{\int_{0}^{1}\left(\sqrt{1-t^{2}}-t+1\right) d t}=\frac{\frac{5}{6}}{\frac{\pi}{4}+\frac{1}{2}} \\
& =\frac{10}{3(\pi+2)} \frac{1}{\int_{0}^{1}\left(\sqrt{1-t^{2}}-t+1\right) d t} \int_{0}^{1} \int_{x-1}^{\sqrt{1-x^{2}}}\left(x^{2}+y^{2}\right) d y d x \\
& \leq \frac{1}{2}\left[\frac{3 \pi-4}{3(\pi+2)}(F(0, g(0))+F(0, h(0)))+\frac{10}{3(\pi+2)}(F(1, g(1))+F(1, h(1)))\right] .
\end{aligned}
$$

So,

$$
\frac{4}{\pi+2} \int_{0}^{1} \int_{x-1}^{\sqrt{1-x^{2}}}\left(x^{2}+y^{2}\right) d y d x \leq 1
$$

Example 4.3 Let $F(x, y)=x^{2}+y^{2}$ and $\triangle$ be bounded by $g(x)=x+2, h(x)=x^{2}$ on $[-1,2]$. Then $g-h$ is not decreasing on $[-1,2]$ and also $F(x, h(x))=x^{2}+x^{4}$ is not convex on $[-1,2]$. So, $g, h$ and $F$ do not hold in the hypothesis of Theorems 3.2 and 3.3. But we have

$$
\frac{g(x)-g(-1)}{x+1}-g^{\prime}(x)=\frac{x+1}{x+1}-1=0, \quad \frac{h(x)-h(-1)}{x+1}-h^{\prime}(x)=-x-1 \leq 0
$$

and

$$
\frac{\partial F(x, g(x))}{\partial g}=2(x+2), \quad \frac{\partial F(x, h(x))}{\partial h}=2 x^{2} .
$$

So,

$$
\begin{aligned}
& \frac{\partial F(x, g(x))}{\partial g}\left[\frac{g(x)-g(-1)}{x+1}-g^{\prime}(x)\right]+\frac{\partial F(x, h(x))}{\partial h}\left[\frac{h(x)-h(-1)}{x+1}-h^{\prime}(x)\right] \\
& =2 x^{2}(-x-1)=-2 x^{2}(x+1) \leq 0 .
\end{aligned}
$$

Thus, we can apply Theorem 3.4

$$
\begin{aligned}
& \frac{1}{\int_{-1}^{2}\left(x+2-x^{2}\right) d x} \int_{-1}^{2} \int_{x^{2}}^{x+2}\left(x^{2}+y^{2}\right) d y d x \\
& \leq \frac{1}{2}\left[\frac{(2-\alpha(2))(F(-1, g(-1))+F(-1, h(-1)))+(\alpha(2)+1)(F(2, g(2))+F(2, h(2)))}{2-(-1)}\right], \\
& \alpha(2)=\frac{\int_{-1}^{2} t\left(t+2-t^{2}\right) d t}{\int_{-1}^{2}\left(t+2-t^{2}\right) d t}=\frac{\frac{9}{4}}{\frac{9}{2}}=\frac{1}{2}, \\
& g(-1)=h(-1)=1, \quad g(2)=h(2)=4 .
\end{aligned}
$$


Hence,

$$
\frac{2}{9} \int_{-1}^{2} \int_{x^{2}}^{x+2}\left(x^{2}+y^{2}\right) d y d x \leq 11
$$

Example 4.4 Let $F(x, y)=x y$ and $\triangle$ be bounded by $g(x)=x+2$, and $h(x)=x^{2}$ on $[-1,2]$. Then $F$ is not convex on $\triangle$, but it is convex relative to $y$, we have

$$
F(x, g(x))=x^{2}+2 x \text { and } F(x, h(x))=x^{3} .
$$

So,

$$
F^{\prime}(x, g(x))+F^{\prime}(x, h(x))=2 x+2+3 x^{2}>0 \text {. }
$$

Hence, by Theorem 3.5, we have

$$
\frac{1}{\int_{-1}^{2}\left(x+2-x^{2}\right) d x} \int_{-1}^{2} \int_{x^{2}}^{x+2} x y d y d x \leq \frac{1}{2}[F(2, g(2))+F(2, h(2))] .
$$

Hence,

$$
\frac{2}{9} \int_{-1}^{2} \int_{x^{2}}^{x+2} x y d y d x \leq 8
$$

\section{Competing interests}

Authors declare that they have no competing interest.

\section{Authors' contributions}

Both the authors contributed equally in preparation as well as in typing and further both authors read the proof and approved the modifications.

\section{Author details}

${ }^{1}$ Department of Mathematics, Faculty of Mathematical Sciences and Computer, Tarbiat Moallem University, 599 Taleghani Avenue, Tehran, 15618, Iran. ${ }^{2}$ Department of Mathematics and Institute of Mathematical Research, Universiti Putra Malaysia (UPM), Serdang, Selangor 43400 UPM, Malaysia.

\section{Acknowledgements}

The authors express their sincere thanks to the referees for the careful and detailed reading of the manuscript and very helpful suggestions that improved the manuscript substantially. The authors also gratefully acknowledge that this research was partially supported by the University Putra Malaysia under the Research University Grant Scheme 05-01-09-0720RU.

Received: 23 August 2012 Accepted: 3 January 2013 Published: 22 January 2013

\section{References}

1. Fejér, L: Über die Fourierreihen, II. Math. Naturwiss. Anz Ungar. Akad. Wiss. 24, 369-390 (1906) (In Hungarian)

2. Yang, GS, Tseng, KH: On certain integral inequalities related to Hermite-Hadamard inequalities. J. Math. Anal. Appl. 239, 180-187 (1999)

3. Dragomir, SS, M Pearce, CE: Selected Topics on Hermite-Hadamard Inequalities. RGMIA Monographs, Victoria University (2000)

4. Dragomir, SS: On the Hadamard's inequality for convex function on the co-ordinated in a rectangle from the plane. Taiwan. J. Math. 5, 775-788 (2001)

5. Matejika, L: Elementary proof of the left multidimensional Hermite-Hadamard inequality on certain convex sets. J. Math. Inequal. 4(2), 259-270 (2010)

6. Zabandan, G, Kılıçman, A: A new version of Jensen's inequality and related results. J. Inequal. Appl. 2012, 238 (2012). doi:10.1186/1029-242X-2012-238 